\title{
A hybridized mixed finite element domain decomposed method for two dimensional magnetotelluric modelling
}

\author{
F. I. Zyserman ${ }^{1}$, L. Guarracino ${ }^{2}$, and J. E. Santos ${ }^{2,3}$ \\ ${ }^{1}$ Depto. de Física, Fac. de Cs. Exactas, U.N.L.P., Calle 49 y 115, 1900 - La Plata, Argentina \\ ${ }^{2}$ CONICET, Depto. de Geofísica Aplicada, Fac. de Cs. Astronómicas y Geofísicas, U.N.L.P., Paseo del Bosque s/n, 1900 - La Plata, Argentina \\ ${ }^{3}$ Department of Mathematics, Purdue University, W. Lafayette, IN, 47907, U.S.A.
}

(Received April 13, 1998; Revised December 18, 1998; Accepted February 16, 1999)

\begin{abstract}
A numerical algorithm to solve the 2D forward problem in magnetotellurics is presented. The method solves Maxwell's equations as a first order system of partial differential equations employing an iterative hybridized mixed domain decomposed finite element procedure. Absorbing boundary conditions are used on the artificial boundaries, diminishing undesired reflection effects and allowing the use of substantially smaller computational domains. Although the algorithm presented can be implemented on both serial and parallel computers, its capabilities are fully utilized on the latters. Results obtained on an IBM SP/2 parallel supercomputer of Purdue University are shown. Also the accuracy of the numerical method is verified by comparison with both numerical and analytical solutions provided by well known methods.
\end{abstract}

\section{Introduction}

The objective of this work is to present a novel numerical procedure to solve the $2 \mathrm{D}$ direct problem in magnetotellurics. Assuming that the earth can be modelled as a horizontallylayered body with two-dimensional inhomogeneities, the numerical algorithm to be defined and analyzed will allow us to obtain approximations to the scattered electromagnetic field generated by anomalies of arbitrary shape in the earth conductivity.

Numerical methods to solve the direct method in magnetotellurics have been proposed by several authors during the last two decades. In a classical work by Wannamaker et al. (1987), a Finite Element (FE) method was employed to solve the problem analyzed here using the standard formulation of Maxwell's equations as a set of two secondorder elliptic equations for the scattered magnetic and electric fields. However, this formulation does not allow discontinuities in the conductivity coefficient, introducing an unnecessary smoothness requirement and numerical complexity. The associated algebraic problem was solved using a standard Gaussian elimination procedure.

In another paper, using also the second-order formulation of Maxwell's harmonic (frequency domain) equations, Travis and Chave (1989), proposed a moving FE procedure to solve the two-dimensional magnetotelluric problem, with a modified Gaussian elimination procedure to solve the associated algebraic problem. Lee and Morrison (1985) gave a solution of the harmonic Maxwell's equations with application to magnetotelluric modelling; and more recently $\mathrm{Pu}$ et al. (1993), Weaver et al. (1996) and Aprea et al. (1997) have also tackled some of the unsolved problems with which the

Copy right(c) The Society of Geomagnetism and Earth, Planetary and Space Sciences (SGEPSS); The Seismological Society of Japan; The Volcanological Society of Japan; The Geodetic Society of Japan; The Japanese Society for Planetary Sciences. researchers in this area are still challenged.

In this work we present a numerical procedure to solve the harmonic Maxwell's equations in its original form as a first-order system of partial differential equations for the electric and magnetic fields. This approach allows us to handle discontinuities in the conductivity coefficient without introducing unnecessary numerical complexity. Absorbing boundary conditions (Sheen, 1997) are used on the artificial borders, which reduces significantly computation times because smaller domains can be considered without loosing accuracy in the solution. Though this algorithm can be tackled without any problem by serial machines, it is specially appropriated to be used on machines capable of parallel processing, and in the present effort we present results obtained on the IBM SP/2 supercomputer at Purdue University. This feature makes this method a very powerful tool when inverting magnetotelluric data, allowing to efficiently handle large amounts of parameters and data.

The iterative hybridized mixed finite element domain decomposition procedure presented here has been presented and analyzed by Santos (1998). The procedure is closely related to the procedure presented by Douglas et al. (1993) for the approximate solution of second-order elliptic problems, and uses the hybridization of mixed finite element methods introduced by Fraeijs de Veubeke $(1965,1975)$ and the domain decomposition method described by Després et al. (1992).

The organization of the paper is as follows. In Section 2 we describe the physical problem and the differential equations and boundary conditions employed for its mathematical description. In Section 3 we introduce the domain decomposition technique and the hybridization method, which are used to give our algorithm its final form. In Section 4 the numerical implementation is shown, and also some of the features of the method are discussed. We devote Section 5 to 
present the results obtained and the efficiency of the parallel algorithm. Finally, in Section 6 we draw the conclusions.

\section{The Forward Differential Model}

Recall that if $\mathbf{E}$ and $\mathbf{H}$ denote, respectively, the electric and magnetic fields for a given angular frequency $w$, then the harmonic Maxwell's equations state that

$$
\begin{aligned}
& \nabla \times \mathbf{H}=\sigma \mathbf{E}, \\
& \nabla \times \mathbf{E}=-i w \mu \mathbf{H},
\end{aligned}
$$

where $\sigma$ and $\mu$ denote the electrical conductivity and magnetic permeability respectively, and as usual in magnetotellurics displacement currents have been neglected. Also, associated to Eqs. (2.1) we have the consistency conditions imposing the continuity of the tangential electric and magnetic fields and the continuity of the current density and magnetic flux normal to any interior interface.

Let us consider Eqs. (2.1) in a two-dimensional rectangular domain $\Omega=\left[0, x_{0}\right] \times\left[0, z_{0}\right]$. The uppermost layer of $\Omega$ represents the air, with a very low conductivity, and the other layers represent a horizontally-layered earth with an embedded cylindrical inhomogeneity as shown in Fig. 1, with the $y$-axis being the symmetry axis.

The electrical conductivity distribution is

$$
\sigma(x, z)= \begin{cases}\sigma_{p}(z) & \text { in } \Omega-\Omega_{s}, \\ \sigma_{p}(z)+\sigma_{s}(x, z) & \text { in } \Omega_{s} \text { (inhomogeneity). }\end{cases}
$$

Assuming that both sources and boundary conditions are also independent of $y$, it is well known that the electromagnetic response in the considered region can be described by two uncoupled electromagnetic modes; the $T E$-mode involving field components $\left(H_{x}, E_{y}, H_{z}\right)$ and the $T M$-mode involving field components $\left(E_{x}, H_{y}, E_{z}\right)$. In what follows, we will analyze in detail the latter, and in Appendix we will briefly indicate the corresponding formulation for the former.

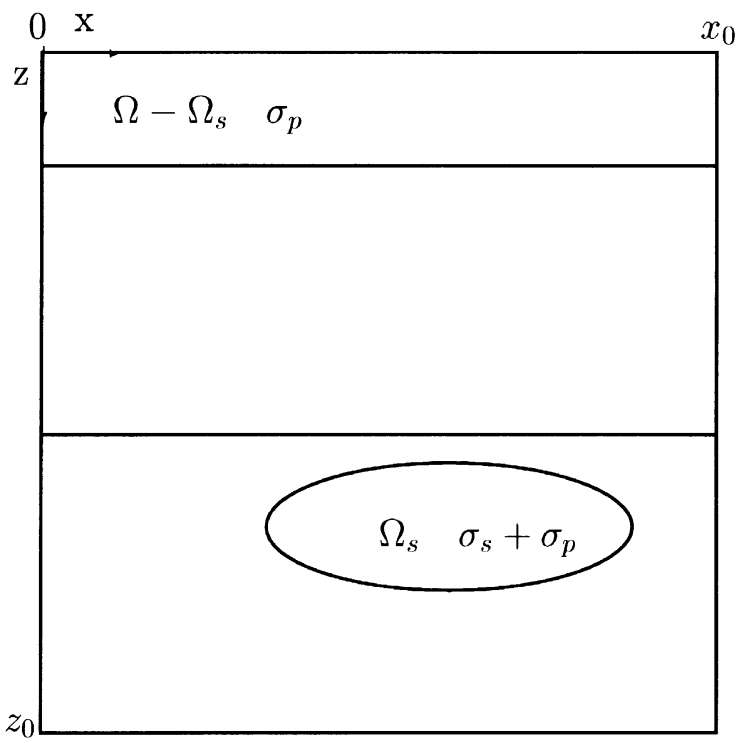

Fig. 1. The two-dimensional model. The symmetry axis $y$ points normally out of the page.
For the $T M$-mode, Eqs. (2.1) yield:

$$
\begin{aligned}
& \frac{\partial H_{y}}{\partial z}=-\sigma E_{x}, \\
& \frac{\partial H_{y}}{\partial x}=\sigma E_{z},
\end{aligned}
$$

and

$$
\frac{\partial E_{x}}{\partial z}-\frac{\partial E_{z}}{\partial x}=-i w \mu H_{y} .
$$

Let us further assume that the incident electromagnetic field is of the form $\boldsymbol{E}_{0}=\left(E_{0 x}, 0,0\right), \boldsymbol{H}_{0}=\left(0, H_{0 y}, 0\right)$. Then the solution of Eqs. (2.3) and (2.4) in $\Omega$ with $\sigma=\sigma_{p}(z)$ (i.e., a horizontally layered earth with no inhomogeneity) are the electric and magnetic fields $\mathbf{E}_{p}(z)=\left(E_{x p}(z), 0,0\right)$ and $\mathbf{H}_{p}(z)=\left(0, H_{y p}(z), 0\right)$, respectively, having $E_{x p}(z)$ and $H_{y p}(z)$ closed analytic expressions. These solutions are the so-called primary solutions (Hohmann, 1983).

Following Coggon (1971) we then define the residual (or scattered) electric and magnetic fields $\tilde{\mathbf{U}}$ and $\tilde{\mathbf{V}}$, respectively, as follows:

$$
\begin{aligned}
\tilde{\mathbf{U}} & =\mathbf{E}-\mathbf{E}_{p}=\left(U_{x}, 0, U_{z}\right), \\
\tilde{\mathbf{V}} & =\mathbf{H}-\mathbf{H}_{p}=\left(0, V_{y}, 0\right)=(0, v, 0),
\end{aligned}
$$

so that

$$
\begin{aligned}
& U_{x}=E_{x}-E_{x p}, \\
& U_{z}=E_{z}, \\
& v=H_{y}-H_{y p} .
\end{aligned}
$$

As we are going to deal with the non-zero components of $\tilde{\mathbf{U}}$ and $\tilde{\mathbf{V}}$, we consider from now on the two-dimensional vector function $\mathbf{U}=\left(U_{x}, U_{z}\right)$ and the scalar function $v$, both defined on the $x z$-plane. Using (2.6), Eqs. (2.3) and (2.4) can be written for the scattered fields in the following way:

$$
\begin{aligned}
& \sigma U_{x}=-\frac{\partial v}{\partial z}-g, \quad \text { with } g=\sigma_{s} E_{x p}, \\
& \sigma U_{z}=\frac{\partial v}{\partial x},
\end{aligned}
$$

and

$$
\frac{\partial U_{x}}{\partial z}-\frac{\partial U_{z}}{\partial x}=-i w \mu v .
$$

Some manipulations on Eqs. (2.7)-(2.8) lead to the well known second order $T M$-equation:

$$
\begin{aligned}
-\nabla \cdot\left(\frac{1}{\sigma} \nabla v\right)+i w \mu v= & -\frac{\partial}{\partial z}\left(\frac{\sigma_{p}}{\sigma}\right) E_{x p} \\
& -i w \mu\left(1-\frac{\sigma_{p}}{\sigma}\right) H_{y p} .
\end{aligned}
$$

Note that the $T M$ equation (2.9) involves derivatives of the conductivity $\sigma$ in the right-hand side. In order to allow for possible discontinuities in the conductivity $\sigma$, we will use a mixed method for Eqs. (2.7)-(2.8) instead of solving (2.9). This choice will also allow us to simultaneously compute the residual electric and magnetic fields $\mathbf{U}$ and $v$ without having to compute the vector field $\mathbf{U}$ numerically once the scalar $v$ is known from (2.9). 
In order to minimize the effect of the artificial boundaries we will use the absorbing boundary condition introduced by Sheen (1997):

$$
(1-i) a \mathbf{U} \cdot \boldsymbol{\tau}+v=0 \quad \text { on } \partial \Omega
$$

where $a=\left(\frac{\sigma}{2 w \mu}\right)^{\frac{1}{2}}, \tau=\left(\tau_{x}, \tau_{z}\right)$ is the unit (clockwise) tangent vector to $\partial \Omega$ the boundary of the domain $\Omega$, and $\mathbf{U} \cdot \boldsymbol{\tau}=U_{x} \tau_{x}+U_{z} \tau_{z}$.

Let us briefly comment what Eq. (2.10) means. Our aim is to simulate as close as possible, the vanishing electromagnetic field in infinity, but considering a finite domain. When considering a Dirichlet boundary condition one must extend the domain until the fields are negligible, and this usually means a large computational domain (and therefore a long time, and a big amount of memory). On the other hand, by using Eq. (2.10) we make a field 'arriving' normally to the border to be 'absorbed' by it, i.e. we make it to leave our domain with no reflections.

A preliminary step to evaluate the numerical approximation of the solution of Eqs. (2.7), (2.8) and (2.10) is to formulate them in a weak (or variational) form. In order to do this, we take a real vector function $\psi(x, z)=\left(\psi_{x}, \psi_{z}\right)$ such that $\psi_{x}, \psi_{z}$ and $\frac{\partial \psi_{x}}{\partial z}-\frac{\partial \psi_{z}}{\partial x}$ are square integrable over $\Omega$. We multiply Eq. (2.7a) by $\psi_{x},(2.7 \mathrm{~b})$ by $\psi_{z}$ and integrate over $\Omega$, using integration by parts in the terms involving derivatives of the potential $v$ :

$$
\begin{aligned}
\int_{\Omega} \sigma U_{x} \psi_{x} d \underline{x}= & -\int_{\Omega}\left(\frac{\partial v}{\partial z}+g\right) \psi_{x} d \underline{x} \\
= & \left.\int_{0}^{x_{0}} v \boldsymbol{\psi} \cdot \boldsymbol{\tau}\right|_{z=0}+\left.v \boldsymbol{\psi} \cdot \boldsymbol{\tau}\right|_{z=z_{0}} d x \\
& +\int_{\Omega} v \frac{\partial \psi_{x}}{\partial z} d \underline{x}-\int_{\Omega} g \psi_{x} d \underline{x}, \\
\int_{\Omega} \sigma U_{z} \psi_{z} d \underline{x}= & \int_{\Omega} \frac{\partial v}{\partial x} \psi_{z} d \underline{x} \\
= & \left.\int_{0}^{z_{0}} v \boldsymbol{\psi} \cdot \boldsymbol{\tau}\right|_{x=0}+\left.v \boldsymbol{\psi} \cdot \boldsymbol{\tau}\right|_{x=x_{0}} d z \\
& -\int_{\Omega} v \frac{\partial \psi_{z}}{\partial x} d \underline{x} .
\end{aligned}
$$

We also multiply Eq. (2.8) by a real square integrable scalar function $\varphi(x, z)$ and integrate over $\Omega$ :

$$
\int_{\Omega}\left(\frac{\partial U_{x}}{\partial z}-\frac{\partial U_{z}}{\partial x}\right) \varphi d \underline{x}=-i w \mu \int_{\Omega} v \varphi d \underline{x} .
$$

Let us for vectors $\boldsymbol{\delta}=\left(\delta_{x}, \delta_{z}\right)$ and $\boldsymbol{\gamma}=\left(\gamma_{x}, \gamma_{z}\right)$ denote: $(\boldsymbol{\delta}, \boldsymbol{\gamma})_{\Omega}=\int_{\Omega} \delta_{x} \bar{\gamma}_{x}+\delta_{z} \bar{\gamma}_{z} d \underline{x}$, and $\langle\boldsymbol{\delta}, \boldsymbol{\gamma}\rangle_{\partial \Omega}=\int_{\partial \Omega} \delta_{x} \bar{\gamma}_{x}+$ $\delta_{z} \bar{\gamma}_{z} d S$. Note that these expressions are also valid for scalars, in that case only one term must be considered in the integrals.

Adding (2.11a) and (2.11b) and using the boundary condition (2.10) we can state our problem in variational form as follows:

Find $(\mathbf{U}, v)$ such that

$$
\begin{gathered}
(\sigma \mathbf{U}, \boldsymbol{\psi})_{\Omega}-\left(v, \frac{\partial \psi_{x}}{\partial z}-\frac{\partial \psi_{z}}{\partial x}\right)_{\Omega} \\
+\langle a(1-i) \mathbf{U} \cdot \boldsymbol{\tau}, \boldsymbol{\psi} \cdot \boldsymbol{\tau}\rangle_{\partial \Omega}=-\left(g, \psi_{x}\right)_{\Omega} \\
\left(\frac{\partial U_{x}}{\partial z}-\frac{\partial U_{z}}{\partial x}, \varphi\right)_{\Omega}+(i w \mu v, \varphi)_{\Omega}=0
\end{gathered}
$$

In the next section we will consider solving the mixed TMequations (2.13) using an iterative hybridized mixed domain decomposition procedure.

\section{Solving the Differential Problem}

In a first stage, we introduce the domain decomposition procedure. Let us therefore subdivide our original domain into a grid of non-overlapping rectangular subdomains $\Omega_{j k}=$ $\left[x_{j}, x_{j+1}\right] \times\left[z_{k}, z_{k+1}\right], j=1, \ldots, n x, k=1, \ldots, n z$. Let $\partial \Omega_{j k}$ be the boundary of the subdomain $\Omega_{j k}$ and let $\Gamma_{j k}^{s}$, $s=L, R, B, T$ be the left, right, bottom and top segments that build the boundary $\partial \Omega_{j k}$.

Consider now solving Eqs. (2.7)-(2.8) with the boundary condition (2.10) on each $\Omega_{j k}$, i.e.,

$$
\begin{gathered}
\sigma U_{x_{j k}}=-\frac{\partial v_{j k}}{\partial z}-g \quad \text { in } \Omega_{j k} \\
\sigma U_{z_{j k}}=-\frac{\partial v_{j k}}{\partial x} \quad \text { in } \Omega_{j k} \\
\frac{\partial U_{x_{j k}}}{\partial z}-\frac{\partial U_{z_{j k}}}{\partial x}=-i w \mu v_{j k} \quad \text { in } \Omega_{j k} \\
(1-i) a \mathbf{U}_{j k} \cdot \boldsymbol{\tau}_{j k}+v_{j k}=0 \text { on } \partial \Omega \cap \partial \Omega_{j k} \equiv B_{j k}^{a} .
\end{gathered}
$$

Because of the decomposition of our domain, consistency conditions are to be imposed on all interior boundaries $\Gamma_{j k}^{s}$. ( $\partial \Omega$ is not included in this consideration). The natural ones are the continuity of the tangential component of $\mathbf{U}_{j k}$ and the potential $v_{j k}$, i.e.

$$
\begin{gathered}
v_{j k}=v_{j^{*} k^{*}} \quad \text { on } \Gamma_{j k}^{s}, \\
\mathbf{U}_{j k} \cdot \boldsymbol{\tau}_{j k}+\mathbf{U}_{j^{*} k^{*}} \cdot \boldsymbol{\tau}_{j^{*} k^{*}}=0 \quad \text { on } \Gamma_{j k}^{s},
\end{gathered}
$$

where

$$
\left(j^{*}, k^{*}\right)= \begin{cases}(j-1, k) & \text { for } s=L \\ (j+1, k) & \text { for } s=R \\ (j, k-1) & \text { for } s=B \\ (j, k+1) & \text { for } s=T\end{cases}
$$

For the iterative domain decomposition procedure to be defined below, following Douglas et al. (1993), it is more convenient to introduce an equivalent Robin transmission boundary condition

$$
\begin{aligned}
v_{j k}= & v_{j^{*} k^{*}}-\beta_{j k}^{s}\left(\mathbf{U}_{j k} \cdot \boldsymbol{\tau}_{j k}+\mathbf{U}_{j^{*} k^{*}} \cdot \boldsymbol{\tau}_{j^{*} k^{*}}\right) \\
& \text { on } \Gamma_{j k}^{s}, s=L, R, B, T,
\end{aligned}
$$

where $\beta_{j k}^{s}$ is a complex parameter with positive real part and negative imaginary part. In the next section we describe which values it takes.

To obtain a variational formulation for Eqs. (3.1)-(3.3) we proceed as in the derivation of (2.13), but applying the Robin transmission boundary conditions (3.7) for the interior boundaries, and the absorbing boundary condition (3.3) on $B_{j k}^{a}$.

Taking into account that indexes $j^{*}$ and $k^{*}$ in Eq. (3.7) involve subdomains adjacent to the $\Omega_{j k}$-cell, we get the following iterative algorithm at the differential level:

1. Choose initial values $\left(\mathbf{U}_{j k}^{0}, v_{j k}^{0}\right)$.

2. Compute $\left(\mathbf{U}_{j k}^{n+1}, v_{j k}^{n+1}\right)$ as the solution of 


$$
\begin{aligned}
& \left(\sigma \mathbf{U}_{j k}^{n+1}, \boldsymbol{\psi}\right)_{\Omega_{j k}}-\left(v_{j k}^{n+1}, \frac{\partial \psi_{x}}{\partial z}-\frac{\partial \psi_{z}}{\partial x}\right)_{\Omega_{j k}} \\
& -\sum_{s}\left\langle v_{j k}^{n+1}, \boldsymbol{\psi} \cdot \boldsymbol{\tau}_{j k}\right\rangle_{\Gamma_{j k}^{s}} \\
& +\left\langle a(1-i) \mathbf{U}_{j k}^{n+1} \cdot \boldsymbol{\tau}_{j k}, \boldsymbol{\psi} \cdot \boldsymbol{\tau}_{j k}\right\rangle_{B_{j k}^{a}} \\
& =-\left(g, \psi_{x}\right)_{\Omega_{j k}} \text {, } \\
& \left(\frac{\partial U_{x_{j k}}^{n+1}}{\partial z}-\frac{\partial U_{z_{j k}}^{n+1}}{\partial x}, \varphi\right)_{\Omega_{j k}}+\left(i w \mu v_{j k}^{n+1}, \varphi\right)_{\Omega_{j k}}=0, \\
& v_{j k}^{n+1}=v_{j^{*} k^{*}}^{n}-\beta_{j k}^{s}\left(\mathbf{U}_{j k}^{n+1} \cdot \boldsymbol{\tau}_{j k}+\mathbf{U}_{j^{*} k^{*}}^{n} \cdot \boldsymbol{\tau}_{j^{*} k^{*}}\right) \text { on } \Gamma_{j k}^{s} \text {. }
\end{aligned}
$$

It must be noted here that for each cell $\Omega_{j k}$, the right hand side of (3.8c) contains unknowns belonging to its surrounding cells, and they are one iteration level behind, i.e. they are considered data on the current iteration level.

The set of Eqs. (3.8), which constitutes the kernel of our presentation, will be solved approximately using a hybridized mixed Finite Element method.

\section{Numerical Implementation}

In order to simplify the description of the numerical procedure, we use the same partition (uniform rectangular cells) of the domain $\Omega$ for both the domain decomposition, and the FE procedures. As we want to approximate simultaneously two different type of functions $\mathbf{U}$ and $v$ we will employ different FE spaces for each one of them. In each cell $\Omega_{j k}$ we use the following approximations:

$$
\begin{aligned}
& \mathbf{U}_{j k}^{n+1}(x, z) \\
& \simeq U_{j k}^{L, n+1} \boldsymbol{\psi}^{L}(x)+U_{j k}^{R, n+1} \boldsymbol{\psi}^{R}(x) \\
& \quad+U_{j k}^{B, n+1} \boldsymbol{\psi}^{B}(z)+U_{j k}^{T, n+1} \boldsymbol{\psi}^{T}(z), \\
& v_{j k}^{n+1}(x, z) \simeq \hat{v}_{j k}^{n+1} \Theta_{j k}(x, z),
\end{aligned}
$$

where $\hat{v}_{j k}^{n+1}$ and $U_{j k}^{s, n+1}, s=L, R, B, T$; are the complex coefficients to be determined at the iteration level $n+1$.

Also, $\psi^{L}(x)=\left(0, \eta_{j k}^{L}(x)\right), \psi^{R}(x)=\left(0, \eta_{j k}^{R}(x)\right)$, $\boldsymbol{\psi}^{B}(z)=\left(\eta_{j k}^{B}(z), 0\right)$ and $\boldsymbol{\psi}^{T}(z)=\left(\eta_{j k}^{T}(z), 0\right)$ constitute the basis of the FE space used to approximate $\mathbf{U}$ in the cell $\Omega_{j k}$ where

$$
\begin{aligned}
& \eta_{j k}^{L}(x)= \begin{cases}-1+\frac{x-x_{j}}{h x_{j}} & \text { in } \Omega_{j k} \\
0 & \text { elsewhere }\end{cases} \\
& \eta_{j k}^{R}(x)= \begin{cases}\frac{x-x_{j}}{h x_{j}} & \text { in } \Omega_{j k} \\
0 & \text { elsewhere, }\end{cases} \\
& \eta_{j k}^{B}(z)= \begin{cases}-1+\frac{z-z_{k}}{h z_{k}} & \text { in } \Omega_{j k} \\
0 & \text { elsewhere }\end{cases} \\
& \eta_{j k}^{T}(z)= \begin{cases}\frac{z-z_{k}}{h z_{k}} & \text { in } \Omega_{j k} \\
0 & \text { elsewhere }\end{cases}
\end{aligned}
$$

and $h z_{k}=z_{k+1}-z_{k}, h x_{j}=x_{j+1}-x_{j}$.

The choice of linear elements to approximate the scattered electric field is closely related to the fact that we assume the electrical conductivity to be piecewise constant (Santos, 1998). If the electrical conductivity $\sigma$ is known to be smooth, higher order polynomials may be employed, but at the cost of increasing the numerical complexity.

Finally, $\Theta_{j k}(x, z)$ is the characteristic function of the subdomain $\Omega_{j k}$ (i.e. $\Theta_{j k}(x, z)$ is equal to one in $\Omega_{j k}$ and zero outside $\Omega_{j k}$ ), and it is the basis of the FE space employed to approximate $v$ in the cell $\Omega_{j k}$.

Thus in each iteration and in each cell, we approximate $\mathbf{U}$ by an ordered pair of linear functions in $x$ and $z$ respectively, and $v$ by a constant value. In this process the parameters $U_{j k}^{s, n+1}, s=L, R, B, T$ are located at the middle point of the respective boundary segment; the parameter $v_{j k}^{n+1}$ is assumed to be located at the center of the domain $\Omega_{j k}$.

Note that the functions $v_{j k}^{n+1}(x, z)$ in $(4.1 \mathrm{~b})$ are allowed to be discontinuous across each interface $\Gamma_{j k}^{s}$. Consequently, imposing the consistency conditions (3.7) would imply that $v_{j k}^{n+1}(x, z)$ is a constant across the whole domain $\Omega$. Thus we hybridize our problem (Arnold and Brezzi, 1985) by introducing on each domain $\Omega_{j k}$ a set of Lagrange multipliers $\lambda_{j k}^{s, n+1}, s=L, R, B, T$ identified with the (constant) value of $v_{j k}^{n+1}(x, z)$ on each interface $\Gamma_{j k}^{s}$.

In terms of the Lagrange multipliers the consistency conditions (3.8c) become

$$
\lambda_{j k}^{s, n+1}=\lambda_{j^{*} k^{*}}^{s^{*}, n}-\beta_{j k}^{s}\left(\mathbf{U}_{j k}^{n+1} \cdot \boldsymbol{\tau}_{j k}+\mathbf{U}_{j^{*} k^{*}}^{n} \cdot \boldsymbol{\tau}_{j^{*} k^{*}}\right),
$$

where

$$
s^{*}= \begin{cases}R & \text { for } s=L \\ L & \text { for } s=R \\ T & \text { for } s=B \\ B & \text { for } s=T\end{cases}
$$

In order to obtain the algebraic form of (3.8), we replace $\mathbf{U}_{j k}^{n+1}$ and $v_{j k}^{n+1}$ in these equations by their approximations given in Eq. (4.1), and taking the test function $\varphi=\Theta_{j k}(x, z)$ in Eq. (3.8b) we obtain

$$
\begin{gathered}
h z_{k}\left(U_{j k}^{L, n+1}+U_{j k}^{R, n+1}\right)+h x_{j}\left(U_{j k}^{B, n+1}+U_{j k}^{T, n+1}\right) \\
+i w \mu h z_{k} h x_{j} \hat{v}_{j k}^{n+1}=0 .
\end{gathered}
$$

Next, we take the test function $\boldsymbol{\psi}=\boldsymbol{\psi}^{L}(x)$ in Eq. (3.8a) and use the trapezoidal rule to calculate the first term of the left hand side, and derive the relation

$$
\begin{aligned}
U_{j k}^{L, n+1}( & \left.\frac{1}{2} \sigma_{j k} h x_{j}+(1-i) a_{j k} \delta_{j 1}\right) \\
& +\lambda_{j k}^{L, n+1}\left(1-\delta_{j 1}\right)-\hat{v}_{j k}^{n+1}=0 .
\end{aligned}
$$

The $\delta_{m n}$ denotes the Kronecker delta, used to include in a single equation terms associated with the boundary $\partial \Omega$ and interior interfaces. From Eq. (4.3) we see that

$$
\lambda_{j k}^{L, n+1}=\lambda_{j-1, k}^{R, n}+\beta_{j k}^{L}\left(U_{j k}^{L, n+1}+U_{j-1, k}^{R, n}\right) .
$$

Using (4.7) in Eq. (4.6) we finally get

$$
\begin{aligned}
& U_{j k}^{L, n+1}\left(1+\beta_{j k}^{L} \zeta_{j k}^{L}\right)-\zeta_{j k}^{L} \hat{v}_{j k}^{n+1} \\
& \quad=-\zeta_{j k}^{L}\left(\beta_{j k}^{L} U_{j-1, k}^{R, n}-\lambda_{j-1, k}^{R, n}\right)\left(1-\delta_{j 1}\right),
\end{aligned}
$$


(a)

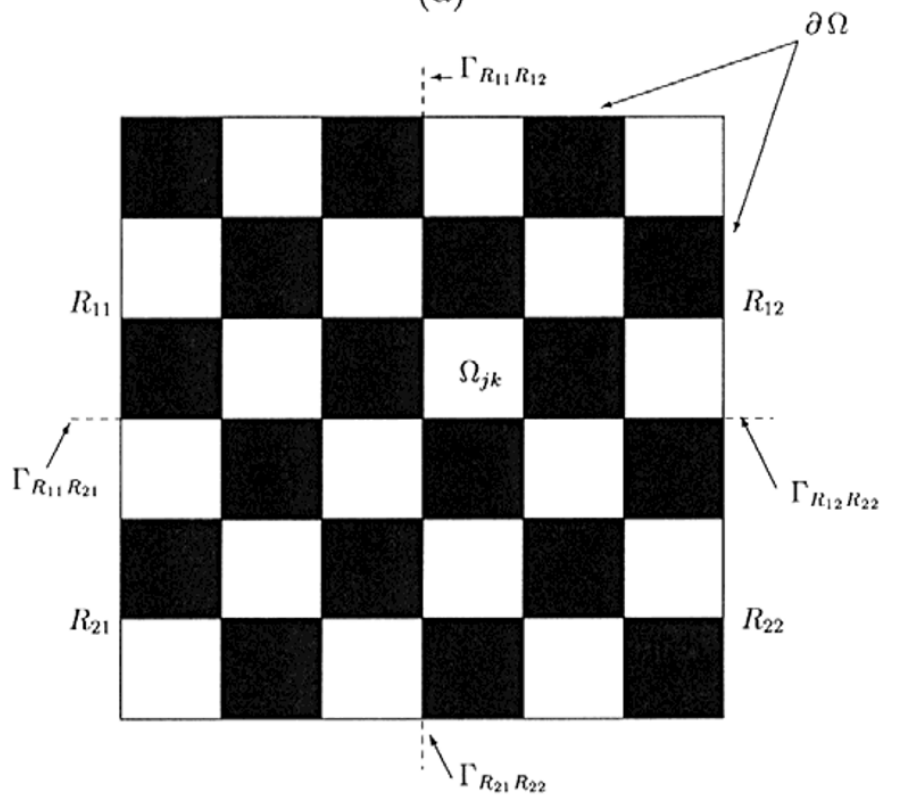

(b)

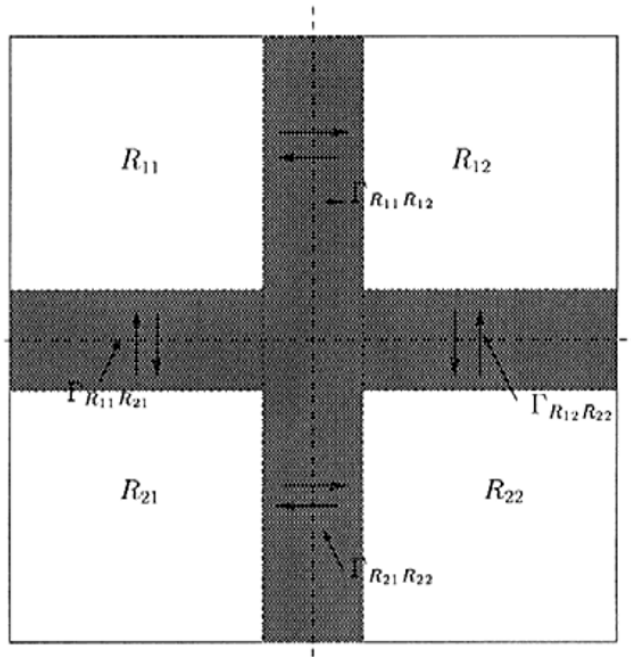

Fig. 2. (a) Scheme for the distribution of the subregions of the domain $\Omega$ on the different processors. The dashed lines represent the boundaries $\Gamma$ of each subregion R; (b) the shaded cells are the ones that are involved in the interchange of information; the arrows show its flow.

with

$$
\zeta_{j k}^{L}=\frac{1-\delta_{j 1}}{\frac{1}{2} \sigma_{j k} h z_{k}+(1-i) a_{j k} \delta_{j 1}} .
$$

Similar expressions to (4.8) are obtained for the coefficients $U_{j k}^{s, n+1}, s=R, B, T$ repeating this procedure with the choice of test functions $\boldsymbol{\psi}^{s}, s=R, B, T$.

Solving Eq. (4.8) for $U_{j k}^{L, n+1}$ and the corresponding equations for the other three coefficients and substituting into (4.5) yields a scalar linear equation involving only the unknown $\hat{v}_{j k}^{n+1}$. Once this equation is solved, i.e. the potential is known, the coefficients $U_{j k}^{s, n+1}, s=L, R, B, T$ are easily obtained from Eq. (4.8) and its other three analogues.

Finally, the Lagrange multipliers $\lambda_{j k}^{s, n+1}, s=L, R, B, T$ are got from Eq. (4.7) and the respective similar ones.

We are now in a position to state our iterative hybridized mixed finite element (MFE) algorithm as follows:

1. Give initial values $U_{j k}^{s, 0}, v_{j k}^{s, 0}, \lambda_{j k}^{s, 0}$ to the unknowns in all cells $\Omega_{j k}$.

2. For all domains $\Omega_{j k}$

- Solve the algebraic set of equations for the unknowns $\hat{v}_{j k}^{n+1}$ and $U_{j k}^{s, n+1}$.

- Compute the Lagrange multipliers $\lambda_{j k}^{s, n+1}$.

3. Check for convergence. If it has not been achieved, go to step 2.

The best results (in the sense of number of iterations and CPU time) were obtained with zero initial values for all the unknowns and with values of $\beta_{j k}^{s}$ given by

$$
\beta_{j k}^{s}=\frac{(1-i)}{2}\left(a_{j k}+a_{j^{*} k^{*}}\right), \quad s=L, R, B, T,
$$

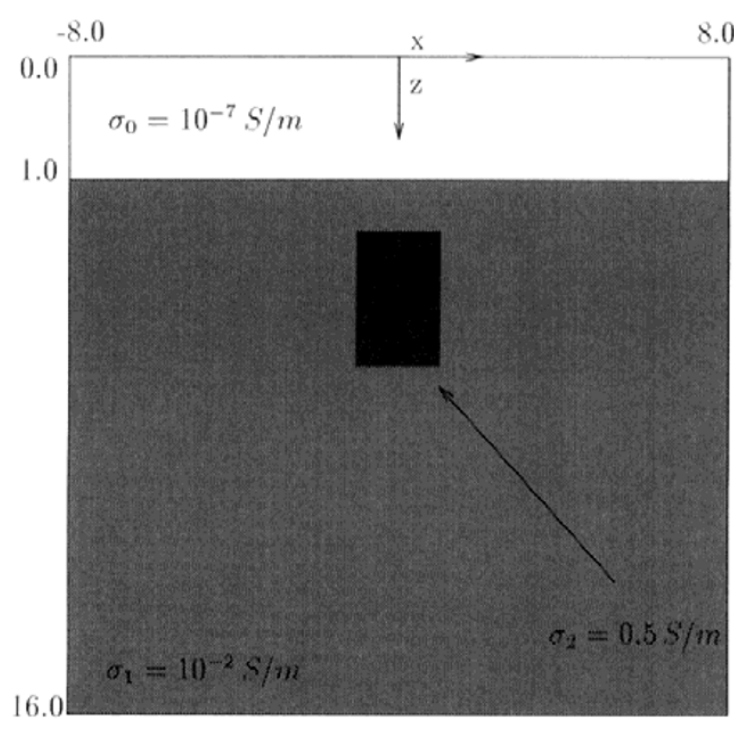

Fig. 3. The first test-model.

where the subscripts $j^{*} k^{*}$ have the same meaning as in (3.6).

The efficiency of the algorithm can be further improved, if in each iteration level instead of solving the algebraic set of equations and updating the Lagrange multipliers one cell after another, we use a white-black scheme (Douglas et al., 1995, 1997).

To do this, the cells in $\Omega$ are divided in two sets, namely white and black ones, in such a way that each white cell is surrounded by black ones (as it happens in a chess-board). The algorithm now reads:

1. Give initial values $U_{j k}^{s, 0}, v_{j k}^{s, 0}, \lambda_{j k}^{s, 0}$ to the unknowns in all 
cells $\Omega_{j k}$.

2. For all white domains $\Omega_{j k}$

- Solve algebraic set of equations for the unknowns $\hat{v}_{j k}^{n+1}$ and $U_{j k}^{s, n+1}$

- Compute the Lagrange multipliers $\lambda_{j k}^{s, n+1}$.

For all black domains $\Omega_{j k}$

- Solve algebraic set of equations for the unknowns $\hat{v}_{j k}^{n+1}$ and $U_{j k}^{s, n+1}$.

- Compute the Lagrange multipliers $\lambda_{j k}^{s, n+1}$.

3. Check for convergence. If it has not been achieved, go to step 2 .

This simple procedure approximately halves the number of iterations needed to converge.

We are now in a position to show the natural way in which this algorithm works on a parallel machine. The most efficient way to perform the calculations is to assign to each
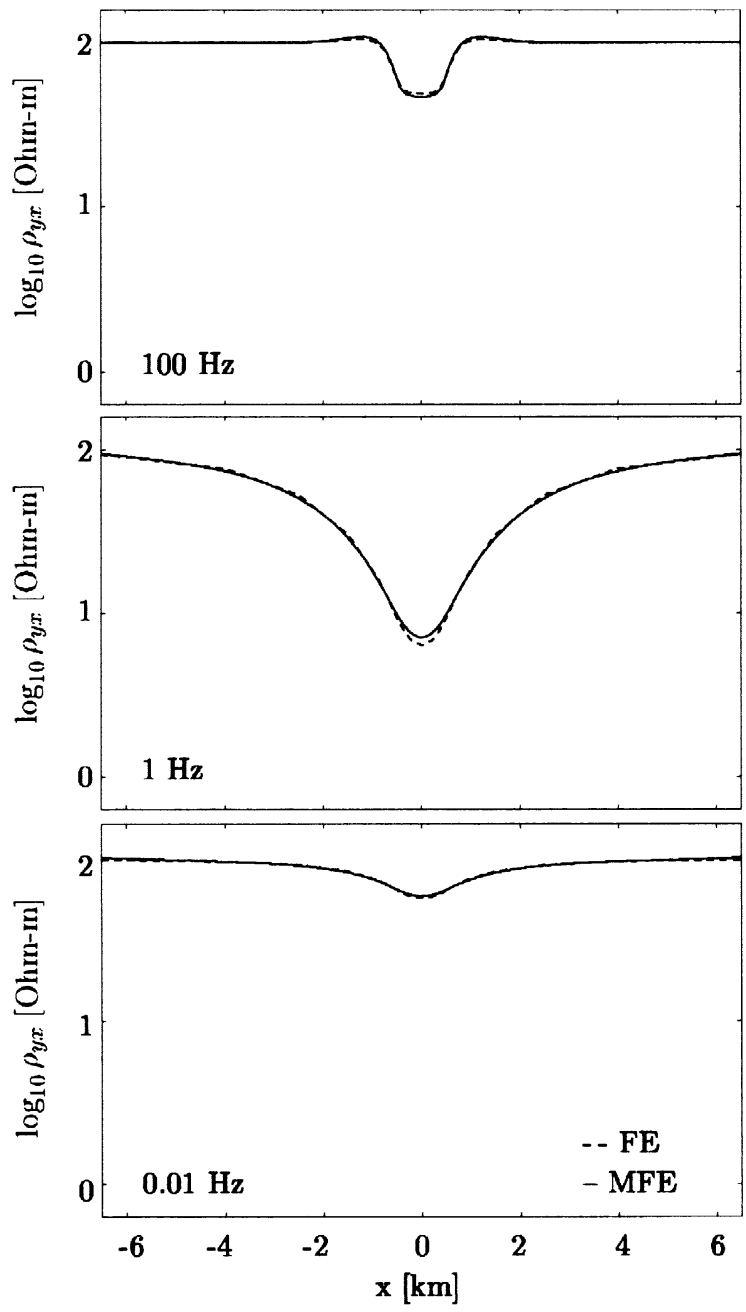

Fig. 4. TE-apparent resistivity $\rho_{y x}$ for different frequencies for the FE method (Wannamaker et al., 1987), and the mixed finite element method (MFE) proposed in this paper. processor, as close as possible, the same number of unknowns (Newman and Alumbaugh, 1997); in our case that means to subdivide our "chess-board" in smaller ones, each containing the same number of cells.

In order to fix ideas, let us work-as displayed in Fig. 2(a) — with the domain $\Omega$ divided in $6 \times 6$ cells, and with four processors.

The processor number 1 solves the MFE algorithm only in $\mathrm{R}_{11}$, and simultaneously the other processors perform their calculations in the respective regions.

The time needed to get the solution is however longer than one fourth of the time with a serial code on one processor. This happens because on each iteration level 'adjacent' processors need to interchange information. For example, in Fig. 2(b) the shaded regions of $\mathrm{R}_{12}$ to the right of $\Gamma_{R_{11} R_{12}}$ and of $\mathrm{R}_{21}$ below $\Gamma_{R_{11} R_{21}}$ involve cells that are neighbours to cells in $\mathrm{R}_{11}$, and therefore, the coefficients $\hat{v}_{j k}^{n+1}$ and $U_{j k}^{s, n+1}$ and Lagrange multipliers $\lambda_{j k}^{s, n+1}$ in all the mentioned shaded regions must be sent to processor number 1 in order to perform adequately step 2 of the proposed algorithm.

Clearly, the same is valid for the other three regions; the
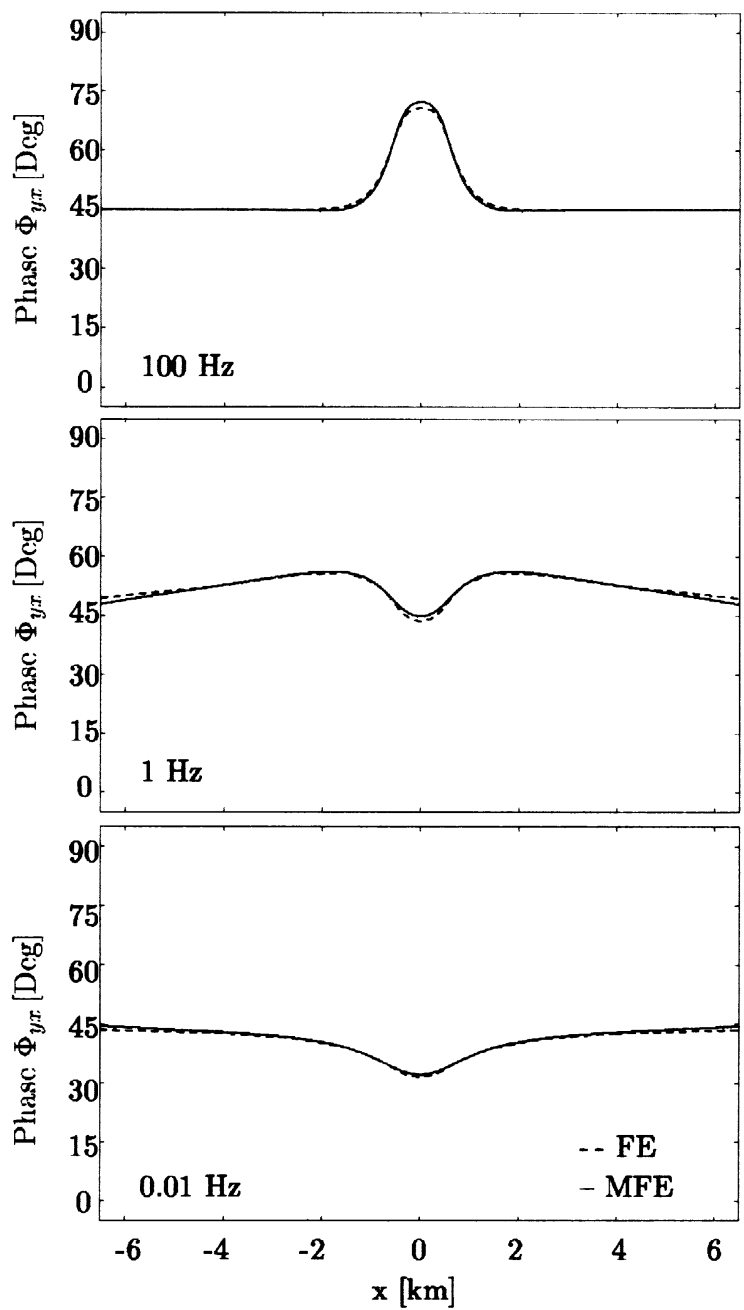

Fig. 5. TE-impedance phase $\Phi_{y x}$ for different frequencies for the FE method (Wannamaker et al., 1987), and the mixed finite element method (MFE) proposed in this paper. 


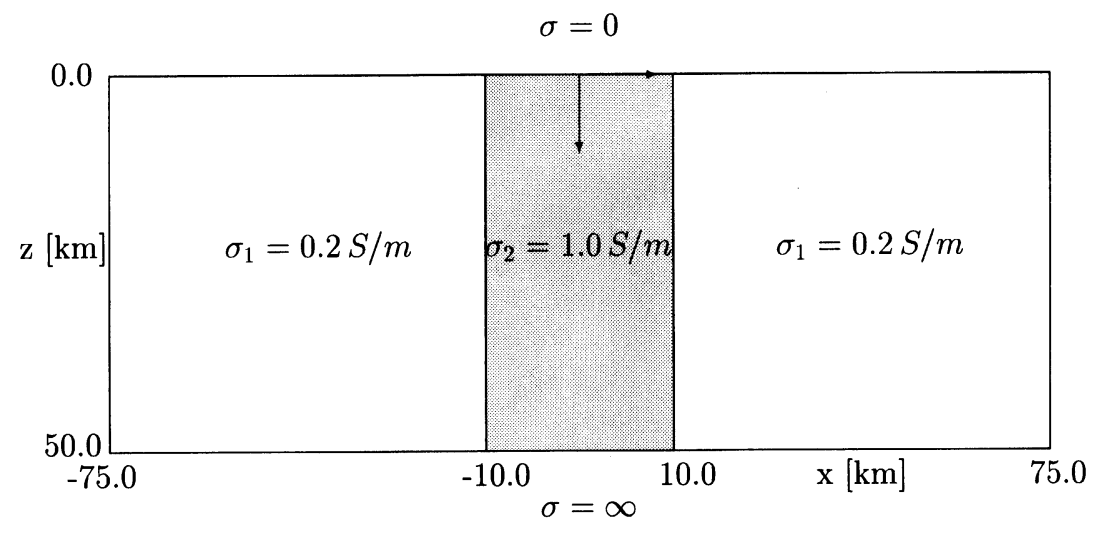

Fig. 6. Second test-model.

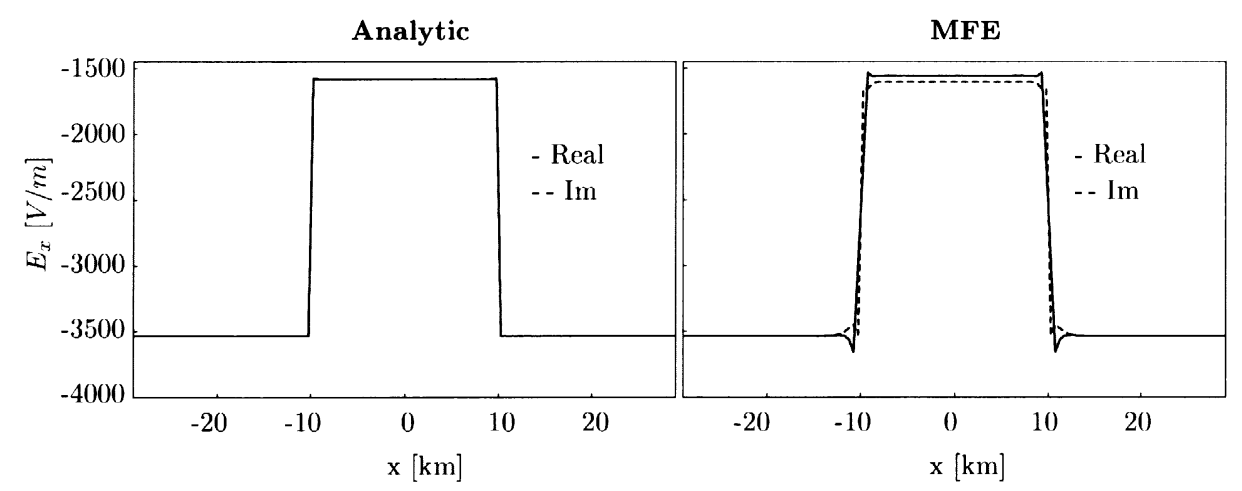

Fig. 7. Real and imaginary parts of the $x$-component of the electric field on the surface, for both the MFE and analytic cases (Weaver et al., 1985), given in units of Volts/m. In the analytic case, both parts are completely superimposed.

in- and outflow of information among processors is done simultaneously twice in the step 2 of the algorithm, after updating the white and black Lagrange multipliers.

The flow of information grows with the number of processors. Therefore, although using more and more processors diminishes monotonically the total time of evaluation, the efficiency of the algorithm decreases when the total number of processors is close to the minimum of $n_{x}$ and $n_{z}$. Clearly this is not an important drawback of the procedure, because this situation is far from being usual.

\section{Example Calculations}

The first case presented is the model proposed by Wannamaker et al. (1987). The geometry chosen is shown in Fig. 3, where a rectangular anomaly of conductivity $\sigma_{2}=0.5 \mathrm{~S} / \mathrm{m}$ and cross section of $1 \mathrm{~km} \times 2 \mathrm{~km}$ lays buried $250 \mathrm{~m}$ in an homogeneous background with conductivity $\sigma_{1}=0.01 \mathrm{~S} / \mathrm{m}$. Because of the absorbing boundary condition, we do not need to extend our computational domain far away from the anomaly. We take for this example a square, with side length of $16 \mathrm{~km}$; this domain is several times smaller than the one used in the cited work.

A requirement of the presented algorithm is to consider a non-zero conductivity for the air region, which was chosen to be $\sigma_{0}=10^{-7} \mathrm{~S} / \mathrm{m}$, and with a height of $1 \mathrm{~km}$.

In order to perform the calculations, we used a $62 \times 82$ grid, (finer grids didn't show different results), and the relative error required to stop the iterative process was $10^{-5}$ (numerical experiments showed that beyond this number the results obtained didn't display any observable change).

Figures 4 and 5 display results for the apparent resistivity $\rho_{y x}$ and impedance phase $\Phi_{y x}$ respectively, corresponding to the above mentioned model and for the $T E$-mode.

In general our results are in very good agreement with that of the referenced work.

Finally we compare our results with the ones provided by the analytical method proposed by Weaver et al. (1985), for the $T M$-mode. The model used is displayed in Fig. 6; a frequency of $1 \mathrm{~Hz}$ was used throughout this case. In order to be able to compare with the analytic results, we modified the top boundary condition, setting $v$ equal to the incident field on the boundary, and using the absorbing boundary condition in the lateral artificial boundaries. The grid size was chosen to be $300 \times 300$, with the same relative error than in the previous example. The algorithm converged after 55 iterations. In Fig. 7 we display the real and imaginary parts of the $x$-component of the electric field on the surface for both the numerical and the analytic methods. For $z=5 \mathrm{~km}$ 

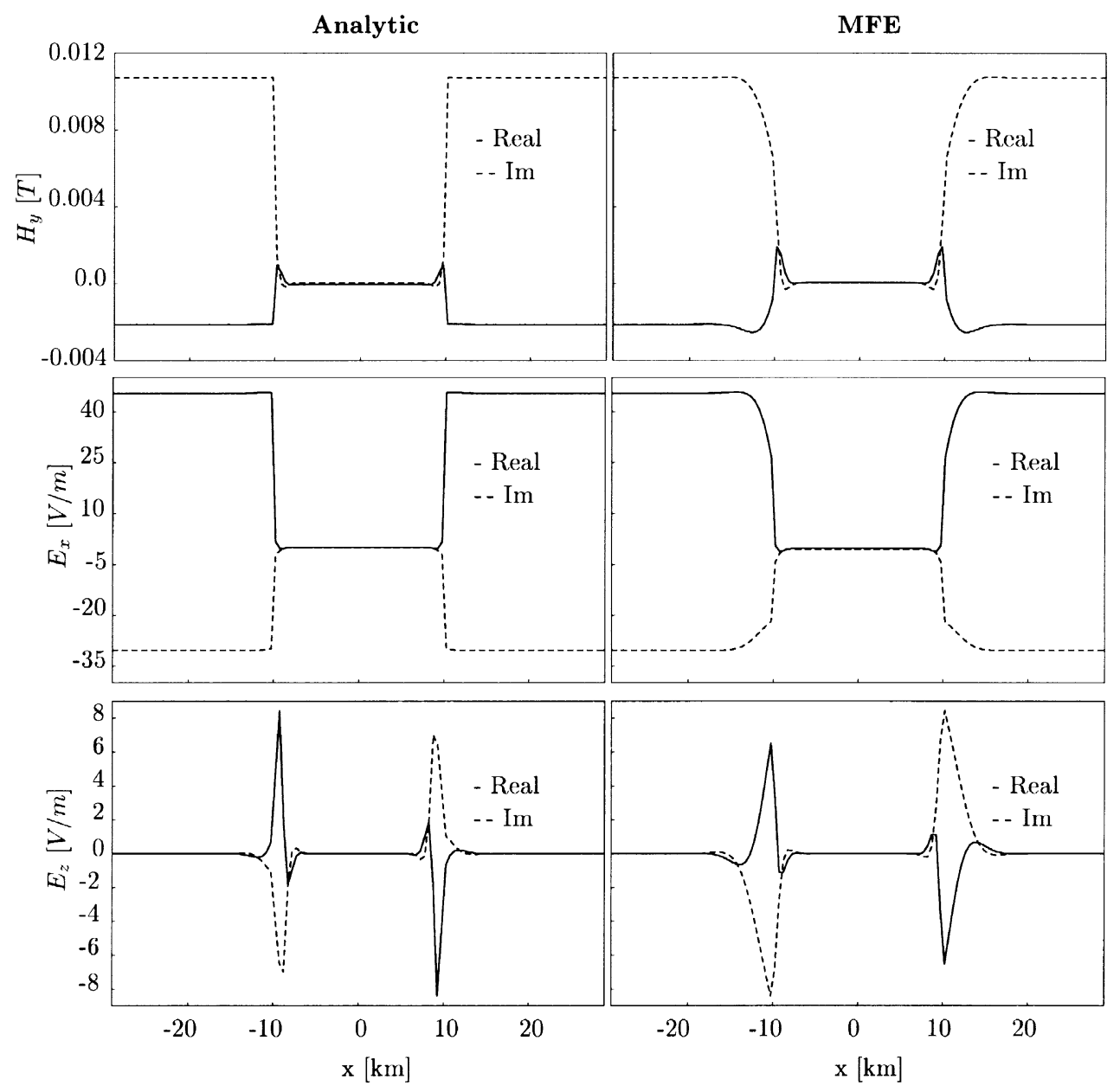

Fig. 8. For the same methods as in Fig. 7, real and imaginary parts of the magnetic field [T], and also both parts for the $x$ - and $z$-components of the electric field [Volts $/ \mathrm{m}]$, for $z=5 \mathrm{~km}$ depth.

Table 1. CPU times for the current example, on an IBM SP/2 parallel supercomputer.

\begin{tabular}{lrrr}
\hline Processors & 4 & 8 & 16 \\
\hline Time $(\mathrm{sec})$ & 52 & 22 & 12 \\
\hline
\end{tabular}

we display in Fig. 8 the real and imaginary parts of the magnetic field, and the real and imaginary parts of the $x$ - and $z$-components of the electric field. The numerical and analytical results are in excellent agreement.

The behaviour of the parallel algorithm is presented in Table 1, which show its performance, for the current example, on an IBM SP/2 parallel at Purdue University. The CPU times displayed were obtained with four, eight and sixteen processors, respectively.

\section{Discussion}

We have presented a numerical algorithm to solve the $2 \mathrm{D}$ forward problem in magnetotellurics. The method solves Maxwell's equations as a first order system of partial differential equations using an iterative hybridized mixed domain decomposed finite element procedure. Absorbing boundary conditions were introduced, which makes it possible to consider smaller computational domains. The algebraic problem associated with this algorithm is much easier to solve than that corresponding to a global FE procedure, since because of the mixed treatment and the domain decomposition technique employed, no global linear system appears, and the procedure reduces to the solution of five independent scalar equations for five unknowns in each cell at the current iteration level. Another advantage of the MFE method is that it requires less data storage than a global FE procedure. The proposed algorithm was implemented on both serial and parallel computers, and results obtained on an IBM SP/2 parallel supercomputer at Purdue University are shown. The method was validated by comparison with analytical results presented previously in the literature.

Acknowledgments. The authors wish to thank Professor P. Wannamaker, for allowing them to use his PW2DI source code to compare his results with the proposed MFE algorithm.

\section{Appendix}

In this section we briefly show the equations we solve when dealing with the $T E$-mode. Recall that in this case we 
have an electric field $\mathbf{E}=\left(0, E_{y}, 0\right)$ and a magnetic field $\mathbf{H}=\left(H_{x}, 0, H_{z}\right)$. Then, from Eqs. (2.1) we obtain

$$
\begin{aligned}
\frac{\partial H_{x}}{\partial z}-\frac{\partial H_{z}}{\partial x} & =\sigma E_{y}, \\
\frac{\partial E_{y}}{\partial z} & =i w \mu H_{x}, \\
\frac{\partial E_{y}}{\partial x} & =-i w \mu H_{z} .
\end{aligned}
$$

As we have already mentioned, expressions for $\mathbf{E}_{p}=$ $\left(0, E_{y p}(z), 0\right)$ and $\mathbf{H}_{p}=\left(H_{x p}(z), 0,0\right)$ induced by an incident wave given by

$$
\boldsymbol{E}_{0}=\left(0, E_{0 y}, 0\right), \quad \boldsymbol{H}_{0}=\left(H_{0 x}, 0,0\right),
$$

into an earth model with conductivity $\sigma_{p}(z)$ can be obtained analytically. Let the residual electric and magnetic fields $\tilde{\mathbf{V}}$ and $\tilde{\mathbf{U}}$ be defined by

$$
\begin{aligned}
\tilde{\mathbf{U}} & =\mathbf{E}-\mathbf{E}_{p}=(0, u, 0)=\left(0, E_{y}-E_{y p}, 0\right), \\
\tilde{\mathbf{V}} & =\mathbf{H}-\mathbf{H}_{p}=\left(V_{x}, 0, V_{z}\right)=\left(H_{x}-H_{x p}, 0, H_{z}\right) .
\end{aligned}
$$

Then, using the same notation as before

$$
\begin{aligned}
& \frac{\partial u}{\partial x}=-i w \mu V_{z}, \\
& \frac{\partial u}{\partial z}=i w \mu V_{x},
\end{aligned}
$$

and

$$
\frac{\partial V_{x}}{\partial z}-\frac{\partial V_{z}}{\partial x}=\sigma v+\left(\sigma-\sigma_{p}\right) E_{y p}=\sigma v+f .
$$

We solve Eqs. (A.4), (A.5) together with the absorbing boundary condition:

$$
a(1-i) u-\mathbf{V} \cdot \boldsymbol{\tau}=0, \quad \text { on } \partial \Omega .
$$

We proceed in the same fashion as before to formulate the mixed weak form of our problem, which can be written as:

$$
\begin{aligned}
&(i w \mu \mathbf{V}, \boldsymbol{\psi})+\left(u, \frac{\partial \psi_{x}}{\partial z}-\frac{\partial \psi_{z}}{\partial x}\right) \\
&+\left\langle\left(\frac{1+i}{2 a}\right) \mathbf{V} \cdot \boldsymbol{\tau}, \boldsymbol{\psi} \cdot \boldsymbol{\tau}\right\rangle=0, \\
&\left(\frac{\partial V_{x}}{\partial z}-\frac{\partial V_{z}}{\partial x}, \varphi\right)-(\sigma u, \varphi)=(f, \varphi) .
\end{aligned}
$$

At this stage, the same steps as proposed in Sections 3 and 4 are followed, with slight variations. When applying the domain decomposition method the consistency conditions on the internal boundaries are the continuity of $u$ and of the tangential component of $\mathbf{V}$. Introducing the Lagrange multipliers associated with the value of the potential $u$ on the interior interfaces $\Gamma_{j k}^{s}$ we can state the Robin transmission boundary condition in the form

$$
\lambda_{j k}^{s}=\lambda_{j^{*} k^{*}}^{s^{*}}+\beta_{j k}^{s}\left(\mathbf{V}_{j k} \cdot \boldsymbol{\tau}_{j k}+V_{j^{*} k^{*}} \cdot \boldsymbol{\tau}_{j^{*} k^{*}}\right) \quad \text { on } \quad \Gamma_{j k}^{S} .
$$

Using the same basis as before for the FE spaces, the numerical algorithm for the $T E$-mode can be stated as:
1. Choose initial values $\left(\mathbf{V}_{j k}^{0}, u_{j k}^{0}, \lambda_{j k}^{0}\right)$.

2. Compute $\left(\mathbf{V}_{j k}^{n+1}, u_{j k}^{n+1}, \lambda_{j k}^{n+1}\right)$ as the solution of

$$
\begin{aligned}
&\left(i w \mu \mathbf{V}_{j k}^{n+1},\right.\boldsymbol{\psi})_{j k}+\left(u_{j k}^{n+1}, \frac{\partial \psi_{x}}{\partial z}-\frac{\partial \psi_{z}}{\partial x}\right) \Omega_{j k} \\
&+\sum_{s}\left\langle\lambda_{j k}^{s, n+1}, \boldsymbol{\psi} \cdot \tau_{j k}\right\rangle_{\Gamma_{j k}^{s}} \\
&+\left\langle\frac{(1+i)}{2 a} \mathbf{V}_{j k}^{n+1} \cdot \boldsymbol{\tau}_{j k}, \boldsymbol{\psi} \cdot \boldsymbol{\tau}_{j k}\right\rangle_{B_{j k}^{a}}=0 \\
&\left(\frac{\partial V_{x_{j k}}^{n+1}}{\partial z}-\right.\left.\frac{\partial V_{z_{j k}}^{n+1}}{\partial x}, \varphi\right) \Omega_{j k}-\left(\sigma u_{j k}^{n+1}, \varphi\right)_{j k}=(f, \varphi) \Omega_{j k}, \\
& \lambda_{j k}^{s, n+1}=\lambda_{j^{*} k^{*}}^{s^{*}, n}+\beta_{j k}^{s}\left(\mathbf{V}_{j k}^{n+1} \cdot \boldsymbol{\tau}_{j k}+\mathbf{V}_{j^{*} k^{*}}^{n} \cdot \boldsymbol{\tau}_{j^{*} k^{*}}\right) .
\end{aligned}
$$

As in the $T M$-case the index $s$ involves all interior boundaries, and $B_{j k}^{a}=\partial \Omega \cap \partial \Omega_{j k}$. For the $T E$-case, the coefficients $\beta_{j k}^{s}=\frac{(1+i)}{2}\left(\frac{1}{a_{j k}}+\frac{1}{a_{j^{*} k^{*}}}\right), s=L, R, B, T$.

\section{References}

Aprea, C., J. R. Booker, and J. Torquil Smith, The forward problem of electromagnetic induction: accurate finite-difference approximations for two-dimensional discrete boundaries with arbitrary geometry, Geophys. J. Int., 129, 29-40, 1997.

Arnold, D. N. and F. Brezzi, Mixed and nonconforming finite element methods: implementation, postprocessing and error estimates, R.A.I.R.O. Modélisation, Mathématique et Analyse Numérique, 19, 7-32, 1985.

Coggon, J. H., Electromagnetic and electrical modelling by the finite element method, Geophysics, 36, 132-155, 1971.

Després, B., P. Joly, and J. E. Roberts, A domain decomposition method for the harmonic Maxwell equations, in Iterative Methods in Linear Algebra, edited by R. Beauwens and P. de Groen, pp. 475-484, Elsevier Science Publishers B. V. (North-Holland), Amsterdam, 1992.

Douglas, J., Jr., P. J. Paes Leme, J. E. Roberts, and J. Wang, A parallel iterative procedure applicable to the approximate solution of second order partial differential equations by mixed finite element methods, Numer. Math., 65, 95-108, 1993.

Douglas, J., Jr., F. Pereira, and L.-M. Yeh, A parallelizable characteristic scheme for two phase flow I: single porosity models, Comp. Appl. Math., 14, 1, 73-96, 1995.

Douglas, J., Jr., F. Hurtado, and F. Pereira, On the numerical simulation of waterflooding of heterogeneous petroleum reservoirs, Comput. Geosci., 1, 155-190, 1997.

Fraeijs de Veubeke, B. X., Displacement and equilibrium models in the finite element method, in Stress Analysis, edited by O. C. Zienkiewicz and G. Holister, pp. 275-284, Wiley, New York, 1965.

Fraeijs de Veubeke, B. X., Stress function approach, in International Congress on the Finite Element Method in Structural Mechanics, pp. 321-332, Bournemouth, 1975.

Hohmann, G. W., Three dimensional EM modelling, Geophys. Surv., 6, 27-54, 1983.

Lee, K. H. and H. F. Morrison, A solution for TM-mode plane waves incident on a two-dimensional inhomogeneity, Geophysics, 50, 466-472, 1985.

Newman, G. and D. Alumbaugh, Three-dimensional massively parallel electromagnetic inversion-I. Theory, Geophys. J. Int., 128, 345-354, 1997.

$\mathrm{Pu}, \mathrm{X}$. H., A. K. Agarwal, and J. T. Weaver, Magnetic field solutions of E-polarization induction problems, J. Geomag. Geoelectr., 45, 859-872, 1993.

Santos, J. E., Global and domain decomposed mixed methods for the solution of Maxwell's equations with application to Magnetotellurics, $\mathrm{Nu}$ merical Methods for Partial Differential Equations, 14, 407-437, 1998.

Sheen, D., Approximation of electromagnetic fields: Part I. Continuous Problems, SIAM J. Appl. Math., 57, 1716-1736, 1997.

Travis, B. J. and A. D. Chave, A moving finite element method for magnetotelluric modelling, Phys. Earth Planet. Inter., 53, 432-443, 1989.

Wannamaker, P. E., J. A. Stodt, and L. Rijo, A stable finite element solution for two-dimensional magnetotelluric modelling, Geophys. J. R. Astr. Soc., 88, 277-296, 1987. 
Weaver, J. T., B. V. Le Quang, and G. Fischer, A comparison of analytic and numerical results for a two dimensional control model in electromagnetic induction.-I. B-polarization calculations, Geophys. J. R. Astr. Soc., 82, 263-277, 1985.

Weaver, J. T., X. H. Pu, and A. K. Agarwal, Improved methods for solving the magnetic field in E-polarization induction problems with fixed and staggered grids, Geophys. J. Int., 126, 437-446, 1996.

F. I. Zyserman (e-mail: zyserman@venus.fisica.unlp.edu.ar), L. Guarracino (e-mail: luisg@fcaglp.fcaglp.unlp.edu.ar), and J. E. Santos (e-mail: santos@math.purdue.edu) 